(1)

(20)

\title{
METS-IR, a novel score to evaluate insulin sensitivity, is predictive of visceral adiposity and incident type 2 diabetes
}

\author{
Omar Yaxmehen Bello-Chavolla,2, Paloma Almeda-Valdes',3, Donaji Gomez-Velasco', \\ Tannia Viveros-Ruiz', Ivette Cruz-Bautista', Alonso Romo-Romo', Daniel Sánchez-Lázaro', \\ Dushan Meza-Oviedo ${ }^{1}$, Arsenio Vargas-Vázquez ${ }^{1,2}$, Olimpia Arellano Campos', Magdalena del Rocío \\ Sevilla-González', Alexandro J Martagón ${ }^{1,4}$, Liliana Muñoz Hernández', Roopa Mehta ${ }^{1}$, \\ César Rodolfo Caballeros-Barragán ${ }^{3}$ and Carlos A Aguilar-Salinas 1,3,4
}

${ }^{1}$ Unidad de Investigación de Enfermedades Metabólicas, Instituto Nacional de Ciencias Médicas y Nutrición Salvador Zubirán, Tlalpan, Mexico, ${ }^{2} \mathrm{MD} / \mathrm{PhD}$ (PECEM) Program, Facultad de Medicina, Universidad Nacional Autónoma de México, Mexico, Mexico, ${ }^{3}$ Department of Endocrinology and Metabolism, Instituto Nacional de Ciencias Médicas y Nutrición Salvador Zubirán, Mexico, Mexico, and ${ }^{4}$ Instituto Tecnológico y de Estudios Superiores de Monterrey Tec Salud, Monterrey, Mexico

Correspondence should be addressed to C A Aguilar-Salinas Email caguilarsalinas@yahoo.com

\begin{abstract}
Objective: We developed a novel non-insulin-based fasting score to evaluate insulin sensitivity validated against the euglycemic-hyperinsulinemic clamp (EHC). We also evaluated its correlation with ectopic fact accumulation and its capacity to predict incident type 2 diabetes mellitus (T2D).

Design and methods: The discovery sample was composed by 125 subjects ( 57 without and 68 with T2D) that underwent an EHC. We defined METS-IR as $\left.\operatorname{Ln}\left(\left(2 * G_{0}\right)+T G_{0}\right) * B M I\right) /(\operatorname{Ln}(H D L-c))\left(G_{0}\right.$ : fasting glucose, $T_{0}$ : fasting triglycerides, BMI: body mass index, HDL-c: high-density lipoprotein cholesterol), and compared its diagnostic performance against the M-value adjusted by fat-free mass (MFFM) obtained by an EHC. METS-IR was validated in a sample with EHC data, a sample with modified frequently sampled intravenous glucose tolerance test (FSIVGTT) data and a large cohort against HOMA-IR. We evaluated the correlation of the score with intrahepatic and intrapancreatic fat measured using magnetic resonance spectroscopy. Subsequently, we evaluated its ability to predict incident T2D cases in a prospective validation cohort of 6144 subjects.

Results: METS-IR demonstrated the better correlation with the MFFM $(\rho=-0.622, P<0.001)$ and diagnostic performance to detect impaired insulin sensitivity compared to both EHC (AUC: $0.84,95 \% \mathrm{Cl}: 0.78-0.90$ ) and the SI index obtained from the FSIVGTT (AUC: $0.67,95 \% \mathrm{Cl}: 0.53-0.81$ ). METS-IR significantly correlated with intravisceral, intrahepatic and intrapancreatic fat and fasting insulin levels $(P<0.001)$. After a two-year follow-up, subjects with METS-IR in the highest quartile (>50.39) had the highest adjusted risk to develop T2D (HR: 3.91, 95\% Cl: 2.25-6.81). Furthermore, subjects with incident T2D had higher baseline METS-IR compared to healthy controls $(50.2 \pm 10.2$ vs
\end{abstract} $44.7 \pm 9.2, P<0.001)$.

Conclusion: METS-IR is a novel score to evaluate cardiometabolic risk in healthy and at-risk subjects and a promising tool for screening of insulin sensitivity.

(C) 2018 European Society of Endocrinology Printed in Great Britain
European Journal of Endocrinology (2018) 178, 533-544
Published by Bioscientifica Ltd. 


\section{Introduction}

Decreased insulin sensitivity, better known as peripheral insulin resistance (IR), is a state of ineffective insulin action in peripheral tissues, which leads to hyperinsulinemia and impaired lipid and glucose homeostasis (1). IR is a risk factor for the development of type 2 diabetes mellitus (T2D), which causes significant health burden world-wide (2). Evaluations of IR often require invasive methods, which has underscored the search for accurate indirect measures of IR (3). Development of accurate and sensitive screening measures to detect IR in early stages to prevent cardiometabolic complications has gained interest $(2,3)$, leading to the development of fasting insulin and noninsulin-based surrogate indexes of IR (4).

Fasting insulin-based indexes, which include the homeostatic model assessment for IR (HOMA-IR) and the quantitative insulin sensitivity check index (QUICKI), have long been employed as the basic evaluations for IR (5). Nevertheless, non-insulin-based fasting IR indexes have been developed to account for the low practicality and variability of insulin-based indexes, substituting insulin measurements for fasting triglycerides, glucose and lipoprotein measures. These include the product of glucose and triglycerides (TyG index), the product of glucose, triglycerides and BMI (TyG-BMI index), and the ratio of triglycerides divided by HDL-c (TG/HDL-c ratio) $(6,7,8)$. However, evaluations of non-insulinbased indices against the gold standard, the euglycemichyperinsulinemic clamp (EHC), offer contrasting results in terms of diagnostic performance and correlation with direct measures of IR (9). In this work, we aimed to generate a non-insulin-based surrogate of insulin action with higher accuracy compared to other insulin and noninsulin-based methods against the EHC and estimated its correlation with pathophysiological components of IR and the metabolic syndrome (MS). Furthermore, we evaluated the capacity of the score to predict incident T2D cases.

\section{Subjects and methods}

\section{Participants and study setting}

In the discovery sample, we included men and women 20-79 years old, with a body mass index (BMI) between 18 and $34.9 \mathrm{~kg} / \mathrm{m}^{2}$ recruited from the outpatient Diabetes Clinic of a university hospital in Mexico City. Subjects with T2D participated if they had a glycated hemoglobin (A1c) concentration $<8 \%$ were not receiving insulin and were treated only with metformin. No subject smoked tobacco, had cardiovascular disease, T2D complications or an acute infection. All biochemical and physiologic evaluations were completed within a month of initial recruitment. Subjects who voluntarily agreed to participate in the study signed an informed consent. The Human Research Ethics Committee of Instituto Nacional de Ciencias Médicas y Nutrición Salvador Zubirán (INCMNSZ) approved the study. All procedures were done in accordance with the Declaration of Helsinki.

\section{Biochemical, insulin sensitivity and body composition evaluations}

\section{Fasting biochemical and anthropometric evaluations}

A complete medical and family history, including use of medications was obtained from all subjects. Subjects were weighed on calibrated scales and height was determined with a floor scale stadiometer; BMI was calculated as weight in $\mathrm{kg}$ divided by the squared product of height in meters.

Blood was obtained between 08:00 and 09:00 h after 8to 12-h fast. Plasma glucose concentration was measured by an automated glucose analyzer (Yellow Springs Instruments, Yellow Springs, OH, USA), serum insulin concentration was measured by using a chemiluminescent immunoassay (Beckman Coulter Access 2), and A1c levels using high-performance liquid chromatography (HPLC) (Variant II Turbo, BIORAD). Lipid concentrations (cholesterol, triglycerides and HDL cholesterol), apo A, apo B, uric acid, creatinine and hepatic enzymes were measured using colorimetric assays (Unicel DxC 600 Synchron Clinical System Beckman Coulter). LDL cholesterol was calculated with the Friedewald equation when triglycerides were $<250 \mathrm{mg} / \mathrm{dL}$.

\section{Euglycemic-hyperinsulinemic clamp and body composition analysis}

We performed a one-stage EHC in subjects who underwent a 12-h fast; subjects with T2D were instructed to suspend treatment three days in advance. The study was not performed if fasting glucose concentrations were $>250 \mathrm{mg} /$ dL. A priming dose of $200 \mathrm{U} / \mathrm{m}^{2} / \mathrm{min}$ of insulin was infused for $5 \mathrm{~min}$, followed by $100 \mathrm{U} / \mathrm{m}^{2} / \mathrm{min}$ for $5 \mathrm{~min}$; subsequently, insulin was infused at a rate of $50 \mathrm{U} / \mathrm{m}^{2}$ body surface area $(\mathrm{BSA}) / \mathrm{min}$. Euglycemia $(\sim 100 \mathrm{mg} / \mathrm{dL})$ was maintained by a variable infusion of $20 \%$ dextrose; arterialized blood samples using a hot box were obtained every 10 min during the final 30 min of theEHC to determine 
glucose and insulin concentrations. Insulin sensitivity was determined by the glucose infusion rate or M-value (10) during the final $30 \mathrm{~min}$ adjusted for fat-free mass (MFFM) obtained by dual X-ray energy absorptiometry (DXA) with a GE Lunar iDXA densitometer. Subcutaneous and intraabdominal adipose tissue volume were quantified using magnetic resonance imaging (MRI); intrapancreatic and intrahepatic triglyceride (IHTG) content was determined using magnetic resonance spectroscopy (11).

\section{Development and validation of the METS-IR index}

\section{Association of fasting biochemical measures with MFFM}

We used linear regression analysis to develop an explanatory model for MFFM using fasting biochemical measures and anthropometric measurements obtained from the day of the EHC. Variables were removed from the model until the best fitting model with the maximum adjusted $R^{2}$ was achieved.

\section{Mathematical modeling}

Standardized beta coefficients for the associated variables in the linear regression model were considered to generate an equation for an IR index. Associated variables with negative beta coefficients were included in the numerator and variables with positive beta coefficient were included in the denominator; the magnitude of the coefficient was used to equilibrate variable contributions within the equation and variables with non-parametric distribution were log-transformed to approximate normality. Finally, we performed algebraic transformations in the model until achieving the equation that produced scores with the higher correlation with MFFM obtained from linear regression analyses, adjusted for age, sex and T2D, which we named metabolic score for IR (METS-IR).

\section{Validation of the METS-IR index against the MFFM from EHC}

For validation of the index, we included 59 additional subjects who also underwent an EHC and body composition analysis using DXA, aged 20-79 years old and $\mathrm{BMI}>30.0 \mathrm{~kg} / \mathrm{m}^{2}$, without diagnosed cardiovascular disease or acute infection and who were evaluated using a 2-h oral glucose tolerance test (GTT) to rule-out T2D. The comparison between the validation and the discovery cohorts (Supplementary Table 1, see section on supplementary data given at the end of this article) showed significant differences between both cohorts; to account for those differences, evaluation was initially carried out in the validation cohort $(n=59)$, followed by evaluation of the overall EHC cohort, including the discovery sample $(n=184)$. We calculated HOMA-IR ((Glucose*Insulin)/405) (12), QUICKI (1/(log insulin + log glucose) ) (13), TyG index (Ln((Glucose* Triglycerides)/2) (6), TG/HDL ratio (triglycerides/HDL-c) (14)) and the TyG*BMI index (TyG-BMI) (15) from fasting biochemical parameters and anthropometric measures in both cohorts. Validation of METS-IR against other surrogate measures of insulin action were evaluated using MFFM values $<25$ th percentile as a cut-off point for IR in our validation cohort.

\section{Validation of METS-IR against the $S I$ index from the modified FSIVGTT and HOMA-IR}

A second validation was carried out in a cohort of 61 subjects, aged 18-55 years old, with BMI between 18.5 and $24.9 \mathrm{~kg} / \mathrm{m}^{2}$, healthy and who were not taking medications that interfere with insulin sensitivity. These subjects were also evaluated with a 2-h oral GTT to rule-out T2D and subsequently insulin sensitivity was assessed with a 3-h modified frequently sampled intravenous GTT (FSIVGTT). Glucose was administered intravenously at a dose of $0.3 \mathrm{~g} / \mathrm{kg}$ for 60 s beginning at time 0 and insulin at a dose of $0.03 \mathrm{IU} /$ $\mathrm{kg}$ at minute 20 for $5 \mathrm{~min}$; blood samples were obtained at $-15,-10,-5,0,2,3,4,5,6,8,10,12,14,16,19,22,24,25$, $27,30,40,50,60,70,90,100,120,140,160$ and $180 \mathrm{~min}$, and data were analyzed using the MINMOD Millennium software to estimate insulin sensitivity (SI index). METS-IR, HOMA-IR, TG/HDL ratio, TyG-BMI and the TyG index were also calculated using fasting laboratory values obtained before the FSIVGTT. A cut-off value for the SI index $<5 \mu \mathrm{U} /$ $\mathrm{min} / \mathrm{mL}$ was defined as IR for this evaluation.

\section{Prediction of T2DM incidence using METS-IR and validation against HOMA-IR}

We then evaluated the capacity of the METS-IR index to predict incident T2D, in our metabolic syndrome cohort, which was developed with the aim to evaluate the risk of MS components in incident T2D, hypertension, and cardiovascular mortality in an urban population living in 9 different cities in Mexico. The inclusion criteria included individuals aged $25-69$ years, $\mathrm{BMI} \geq 23 \mathrm{~kg} / \mathrm{m}^{2}$, without T2D, hypertension or other significant cardiovascular comorbidities and obese individuals $\left(\mathrm{BMI} \geq 30 \mathrm{~kg} / \mathrm{m}^{2}\right.$ ) with at least one of the following conditions: blood pressure $\geq 140 / 90 \mathrm{mmHg}$, fasting glucose $>100 \mathrm{mg} / \mathrm{dL}$, total cholesterol $>200 \mathrm{mg} / \mathrm{dL}$ and triglyceride levels 
$>150 \mathrm{mg} / \mathrm{dL}$. Individuals with diagnosed T2D, coronary artery disease, cerebral vascular disease, alcoholism, taking corticosteroids, with liver disease, kidney dysfunction or life-threatening diseases that would prevent the two-year follow-up were excluded.

Subjects were interviewed to obtain medical history, sociodemographic information, dietary and physical activity habits and anthropometric measurements, including weight, height, and waist circumference. Blood pressure measurement was also performed. We obtained a $20 \mathrm{~mL}$ blood sample after 9- to 12 -h fast to measure of glucose, insulin, total and HDL cholesterol, triglyceride, apolipoprotein B and C-reactive protein concentrations using the same laboratory techniques as described earlier. These same evaluations were carried out after a minimum of two-year follow-up. Incident T2D was defined as a construct of previous medical diagnosis of T2D, taking oral hypoglycemic medication and/or fasting glucose levels as determined by current ADA guidelines. Time to follow-up was estimated from recruitment up to the last follow-up or T2D diagnosis, whichever occurred first. We also performed validation of METS-IR against HOMA-IR using the baseline evaluation of our MS cohort, using scores $>75$ th percentile as the cut-off point to define IR.

\section{Statistical analysis}

Intergroup differences and paired data

To evaluate intergroup differences in sociodemographic, biochemical measures and IR indexes, we used Student's t-test and Mann-Whitney $U$ where appropriate. Frequency distribution of categorical variables is reported as frequencies and percentages and was compared between groups using chi-squared tests. For measurements in follow-up studies, we used Student's paired $t$-test and Wilcoxon's rank-sign tests, where appropriate. Logarithmic transformations were applied to approximate normality in those variables showing a non-parametric distribution. Data are presented as mean \pm S.D. or as median and interquartile range.

\section{Validation of METS-IR}

We used partial correlation analysis to evaluate correlation of individual IR indexes, including METS-IR, against MFFM for the EHC cohort, SI index for the FSIVGTT cohort and HOMA-IR for the MS cohort, adjusted for age, sex and the presence of T2D where appropriate; we generated 95\% confidence intervals for the correlations using bootstrap sampling drawing 2000 random stratified samples. Diagnostic performance was evaluated using areas under the receiving-operating characteristic curve (AUC of ROC) and 95\% confidence intervals were estimated using bootstrap sampling drawing 2000 stratified random samples in both cohorts. To estimate differences between AUC of ROC curves, we performed non-parametric ROC tests using a stratified bootstrap sampling method using the $p R O C$ package from $\mathrm{R}$, version 3.4.3, as proposed by DeLong et al. (16). The cut-off point was determined using the Youden index; sensitivity, specificity, positive and negative predictive values and likelihood ratios (PPV, $\mathrm{NPV}, \operatorname{LR}(+), \operatorname{LR}(-)$, respectively) were calculated using the OptimalCutpoints package from R, version 3.4 .3 (17).

\section{Correlation of METS-IR with pathophysiological components of IR and MS}

To evaluate dose-response correlation of physiologic parameters with METS-IR scores, trend analysis with linear regression was used against quartiles of intrahepatic, intrapancreatic and intravisceral fat adjusted for age, sex and T2D. Intrapancreatic and intrahepatic fat, fasting insulin and body composition measures using DXA were evaluated to develop an explanatory model for METS-IR using linear regression analyses. Variables were removed from until achieving the model with the highest adjusted $R^{2}$ value.

\section{Prediction of incident T2D using METS-IR}

To evaluate the association of the METS-IR score with incident T2D, we performed survival analysis comparing across METS-IR terciles and quartiles using Kaplan-Meier curves compared with log-rank tests. Cox proportionalrisk regression analyses were used to evaluate risk of incident T2D across terciles and quartiles of METS-IR scores adjusted for age, sex, family history of T2D, hypertension, physical activity, waist circumference and smoking. Statistical analyses were performed using the Statistical Package for Social Sciences software (SPSS, version 21.0), R software (Version 3.4.4) and GraphPad Prism, version 6.0.

\section{Results}

\section{Study population}

An outline of the study subjects and phases is presented in Fig. 1. In the discovery sample, we included 67 and 58 subjects with and without T2D, respectively. Subjects without T2D were predominantly female, younger and with a significantly lower BMI, A1c, fasting glucose, insulin and liver enzyme levels compared to subjects 
with T2D $(P<0.001)$. No significant differences were observed in triglyceride, total cholesterol, LDL-c, HDL-c and serum creatinine concentrations. As expected, raw M-values, weight-adjusted M-values, MFFM and QUICKI values were significantly higher for subjects without T2D $(P<0.001)$; conversely, HOMA-IR, TG/HDL, TyG and TyGBMI indexes were lower $(P<0.001$, Table 1$)$.

\section{Mathematical modeling of METS-IR}

In linear regression analyses (Table 2), we identified significant associations between the MFFM and BMI, triglyceride, HDL-c and glucose concentrations $\left(r^{2}=0.309\right.$, $P<0.001)$. Standardized beta coefficients were negative for triglycerides, BMI and glucose $(\beta=-0.170, \beta=-0.186$ and

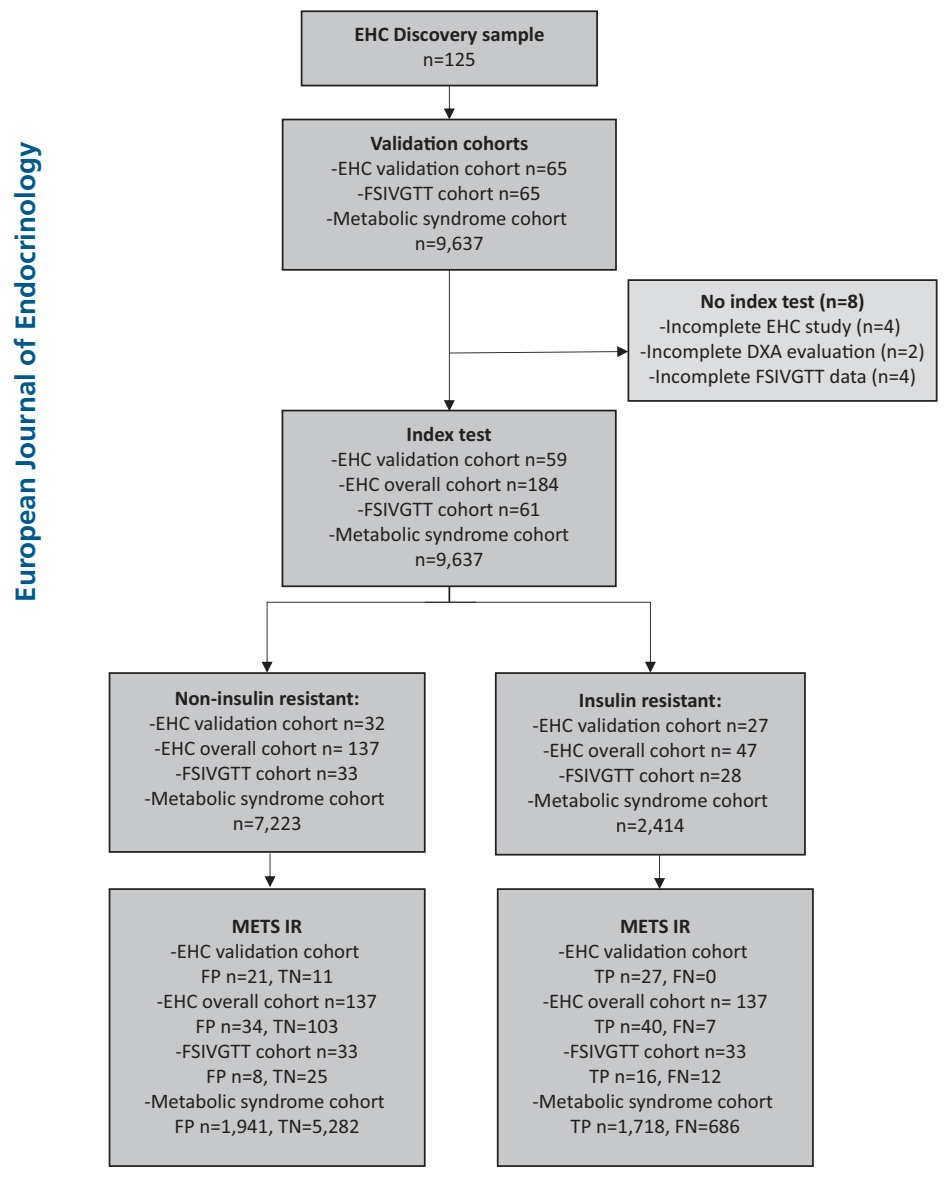

Figure 1

STARD diagram of study phases and populations used for the development, validation and prediction of incident type 2 diabetes with the METS-IR score. EHC, Euglycemichyperinsulinemic clamp; FP, False positive; FN, False negative; FSIVGTT, Frequently sampled intravenous glucose tolerance test; TN, True negative; TP, True positive. $\beta=-0.305$, respectively) but positive for HDL-c $(\beta=0.194)$. Given the near twofold difference in the coefficient for glucose, a twofold multiplier was added to reflect regression coefficients in the equation. Non-parametric variables were log-transformed and algebraic transformations were performed yielding the resulting equation METS-IR $=(\mathrm{Ln}$ $\left.\left(\left(2 * \mathrm{G}_{0}\right)+\mathrm{TG}_{0}\right){ }^{*} \mathrm{BMI}\right) /\left(\mathrm{Ln}\right.$ (HDL-c)), where $\mathrm{G}_{0}$ and $\mathrm{TG}_{0}$ represent fasting glucose and triglyceride concentrations, respectively.

\section{Association of METS-IR and MFFM}

The better correlation of fasting IR indexes and the MFFM was observed for METS-IR $(\rho=-0.569$, Supplementary Table 2), which was slightly higher than the TyG-BMI score $(\rho=-0.555)$. For individuals without T2D, METS-IR also displayed the better correlation with MFFM $(\rho=-0.652)$, followed by TyG-BMI $(\rho=-0.649)$ and HOMA-IR $(\rho=0.646)$. METS-IR had the highest correlation in subjects with T2D ( $\rho=-0.504)$, followed by the TyG-BMI index $(\rho=-0.442)$. In linear regression analysis, METS-IR had a significant association with MFFM $\left(r^{2}=0.376, P<0.001\right)$ adjusted for age, sex and T2D, which was higher than the explanatory model of the independent variables.

\section{METS-IR, visceral adiposity and hyperinsulinemia}

In our discovery sample, we observed a significant correlation between METS-IR and intravisceral $(\rho=0.660$, $P<0.001)$ intrahepatic $\quad(\rho=0.636, \quad P<0.001)$ and intrapancreatic fat $(\rho=0.408, P<0.001)$. These associations were confirmed using linear regression analysis, adjusted by the presence of T2D, age and sex (Fig. 2). In addition, we observed a significant trend of higher METS-IR scores correlated with increasing percentiles of intravisceral, intrahepatic and intrapancreatic fat $(P<0.001$ for all). We found a significant correlation between fasting insulin concentration $(\rho=0.2608, P=0.001)$ and METS-IR score, adjusted by age, sex and the presence of T2D. Using linear regression analysis (Table 3 ), we also observed that fasting insulin, intrahepatic, intravisceral and intrapancreatic fat explain $64.5 \%$ of the variability in METS-IR.

\section{Diagnostic performance and validation of METS-IR against the MFFM}

In the EHC cohort $(N=184)$, we identified 47 subjects with IR using the MFFM value $<25$ th percentile as a cutoff point $(<6.39 \mathrm{mg} / \mathrm{min} / \mathrm{kg}$ FFM), 20 subjects $(16.0 \%)$ from the original cohort and 27 newly included subjects 
Table 1 Sociodemographic variables, fasting biochemical measures, results of EHC and subrogate fasting insulin resistance indexes compared between diabetic and nondiabetic subjects in the discovery sample.

\begin{tabular}{l} 
Parameter \\
\hline Female sex (\%) \\
Age $( \pm$ s.D.) \\
HbA1c $(\%)$ \\
BMI $\left(\mathrm{kg} / \mathrm{m}^{2}\right)$ \\
Fasting glucose $(\mathrm{mg} / \mathrm{dL})$ \\
Fasting insulin $(\mu \mathrm{l} / \mathrm{mL})$ \\
Triglycerides $(\mathrm{mg} / \mathrm{dL})$ \\
Total cholesterol (mg/dL) \\
LDL-c (mg/dL) \\
HDL-c (mg/dL) \\
Serum creatinin (mg/dL) \\
ALT (IU/L) \\
AST (IU/L) \\
GGT (IU/L) \\
M-value (mg/min) \\
M-value adjusted by body mass (mg/min/kg) \\
M-value adjusted by fat-free mass $(\mathrm{mg} / \mathrm{min} / \mathrm{kg})$ \\
HOMA-IR \\
QUICKI \\
TG/HDL \\
TyG \\
TyG-BMI
\end{tabular}

\begin{tabular}{c}
\hline Nondiabetic $(n=57)$ \\
\hline $39(68.4 \%)$ \\
$32.19 \pm 9.75$ \\
$5.20 \pm 0.35$ \\
$26.18 \pm 3.79$ \\
$89.21 \pm 7.67$ \\
$7.60 \pm 4.72$ \\
$104.0(80.0-158.8)$ \\
$174.68 \pm 33.72$ \\
$105.02 \pm 12.15$ \\
$43.91 \pm 9.36$ \\
$0.70 \pm 0.18$ \\
$22.5(17.0-32.3)$ \\
$22.5(18.8-27.3)$ \\
$15.0(11.0-25.3)$ \\
$567.71 \pm 210.86$ \\
$8.22 \pm 2.96$ \\
$13.36 \pm 4.76$ \\
$1.39(1.00-2.33)$ \\
$0.33 \pm 0.04$ \\
$3.13 \pm 2.32$ \\
$8.52 \pm 0.57$ \\
$227.16 \pm 43.03$
\end{tabular}

\begin{tabular}{c}
\hline Diabetic $(n=67)$ \\
\hline $37(54.4 \%)$ \\
$52.97 \pm 12.08$ \\
$6.34 \pm 0.69$ \\
$28.78 \pm 3.34$ \\
$118.07 \pm 30.01$ \\
$10.50 \pm 5.62$ \\
$134.0(106.5-179.5)$ \\
$183.92 \pm 35.67$ \\
$108.47 \pm 26.66$ \\
$45.10 \pm 12.75$ \\
$0.73 \pm 0.21$ \\
$28.0(21.0-39.5)$ \\
$27.0(22.5-34.0)$ \\
$23.0(16.0-30.5)$ \\
$401.45 \pm 154.71$ \\
$5.51 \pm 2.29$ \\
$9.01 \pm 3.91$ \\
$2.70(1.62-4.83)$ \\
$0.36 \pm 0.03$ \\
$4.30 \pm 3.42$ \\
$9.02 \pm 0.58$ \\
$258.23 \pm 38.86$
\end{tabular}

\begin{tabular}{r}
\hline P-value \\
\hline 0.110 \\
$<0.001$ \\
$<0.001$ \\
$<0.001$ \\
$<0.001$ \\
0.002 \\
0.070 \\
0.133 \\
0.566 \\
0.506 \\
0.391 \\
0.009 \\
$<0.001$ \\
$<0.001$ \\
$<0.001$ \\
$<0.001$ \\
$<0.001$ \\
$<0.001$ \\
$<0.001$ \\
$<0.001$ \\
$<0.001$ \\
$<0.001$
\end{tabular}

ALT, Alanine aminotransferase; AST, Aspartate aminotransferase; BMI, Body mass index; EHC, Euglycemic-hyperinsulinemic clamp; HbA1c, Glycated hemoglobin; HDL-c, High-density lipoprotein cholsterol; HOMA-IR, homeostatic model assessment for IR; LDL-c, Low-density lipoprotein cholecterol; QUICKI, quantitative insulin sensitivity check index; TG/HDL, Ratio of triglycerides and high-density lipoprotein cholesterol; TyG, TyG index; TyG-BMI, TyG*BMI index.

(45.8\%). METS-IR had an AUC of 0.845 (95\% CI: 0.783$0.899)$ to identify IR in the combined validation cohort and 0.738 in the EHC validation cohort (95\% CI: 0.601$0.866)$, which did not differ significantly $(P=0.18)$ from the discovery sample (AUC: $0.853,95 \%$ CI: 0.769-0.926, Fig. 3A and B). The AUC was lower for individuals with T2D (AUC $=0.839,95 \%$ CI $0.728-0.931$ ) than for healthy individuals (AUC $=0.852,95 \%$ CI $0.778-0.920$ ) but the difference was not statistically significant $(P=0.833)$. A cut-off value for METS-IR of $>51.13$ had a sensitivity of $85.12 \%$ (95\% CI $71.7-93.8 \%$ ) and a specificity of $75.2 \%$ (95\% CI $67.1-82.2 \%)$ to identify cases of IR diagnosed by MFFM. The NPV and PPV were 93.6\% (95\% CI 86.7$95.7 \%$ ) and $54.0 \%$ (95\% CI 44.2-75.7\%), respectively; the positive and negative likelihood ratios were 3.43 (95\% CI 2.50-4.70) and $0.20(0.10-0.39)$.

Evaluation of other IR indexes using AUC of ROC curves (Table 4) against METS-IR showed the higher AUC for HOMA-IR, followed by METS-IR; among non-insulin fasting indexes, METS-IR had the higher performance, followed by the TyG-BMI index. We observed no significant differences in the AUCs of METS-IR, HOMA-IR and the TyG-BMI index; however, METS-IR had a significantly higher AUC than QUICKI, TyG index and TG/HDL ratio

Table 2 Linear regression analysis showing fasting laboratory values and anthropometric measurements associated to the M-value adjusted by fat-free mass, used for the development of the index.

\begin{tabular}{|c|c|c|c|c|c|}
\hline & Beta & Standardized beta & $\boldsymbol{T}$ & P-value & $95 \% \mathrm{Cl}$ \\
\hline \multicolumn{6}{|l|}{ Variable } \\
\hline Triglycerides & -0.012 & -0.170 & -1.983 & 0.050 & -0.023 to 0.000 \\
\hline HDL-C & 0.081 & 0.194 & 2.433 & 0.016 & 0.015-0.147 \\
\hline $\mathrm{BMI}$ & -0.236 & -0.186 & -2.235 & 0.027 & -0.445 to -0.027 \\
\hline Glucose & -0.054 & -0.305 & -3.751 & $<0.001$ & -0.82 to -0.025 \\
\hline
\end{tabular}

Adjusted for age, sex and the presence of T2D.

95\% Cl, 95\% Confidence interval; BMI, Body mass index; HDL-c, High-density lipoprotein cholsterol; MFFM, M-value adjusted by fat-free mass; T2D, Type 2 diabetes mellitus. 
A
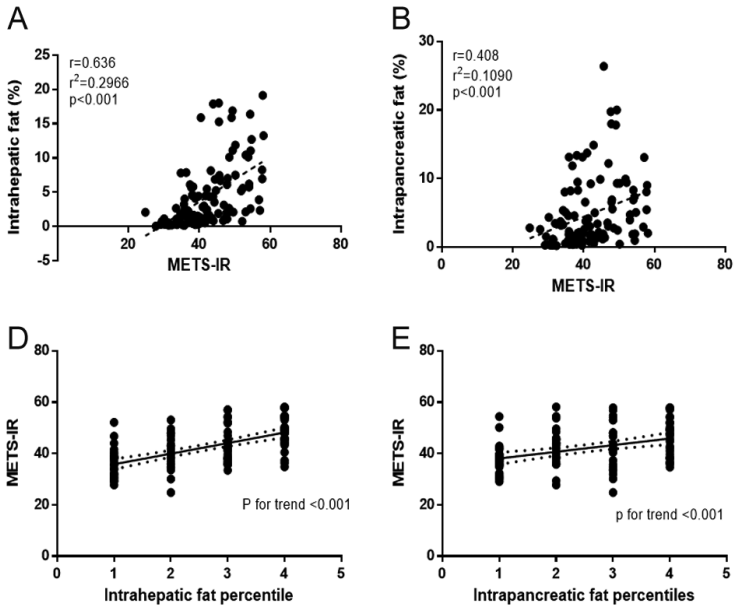

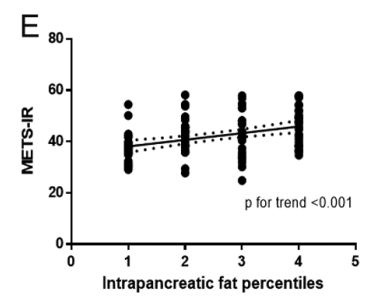

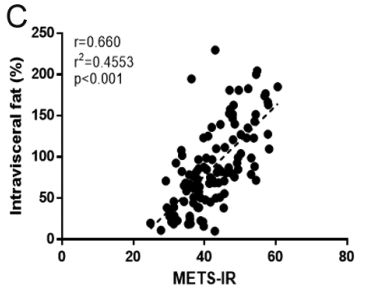

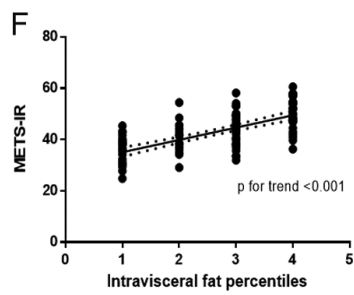

Figure 2

( $A, B$ and $C$ ) show linear regressions and correlations between intrahepatic (A), intrapancreatic (B) and intravisceral (C) fat content, showing positive and significant associations with METS-IR values. Figures $(D, E$ and $F$ ) show the comparison between METS-IR distributions according to intrahepatic (D), intrapancreatic (E) and intravisceral (F) fat content quartiles, showing a statistically significant trend $(P<0.001)$ of increasing METS-IR values with higher intravisceral, intrapancreatic and intrahepatic fat content. in both healthy subjects and the overall EHC cohort $(P<0.01)$. In subjects with T2D, METS-IR and the TyG-BMI index had the best diagnostic performance; we observed no significant differences among AUCs of fasting indexes in subjects with T2D. Cut-off points along with its diagnostic performance indexes showed the higher sensitivity and NPV for METS-IR compared to other non-insulin-based fasting IR surrogates (Supplementary Table 3).

\section{Validation against the modified FSIVGTT and HOMA-IR}

In the second validation cohort against the SI index obtained from the modified FSIVGTT, we evaluated 61 healthy subjects, with an average age of $24.8 \pm 4.4$ years and female predominance (73.8\%). The average SI index was $6.23 \pm 3.58$ and 28 subjects $(45.9 \%)$ were classified with IR. Using partial correlation analysis adjusted by age and sex, all fasting indexes were significantly correlated to the SI index calculated by MINMOD (Supplementary Table 4). The highest AUC of fasting indexes (Fig. 3C) against IR (as defined by an SI index $<5 \mu \mathrm{U} / \mathrm{min} / \mathrm{mL}$ ) was for QUICKI (AUC: 0.68, 95\% CI: 0.54-0.82), followed by METS-IR (0.67; 95\% CI: 0.53-0.81) and the TG/HDL ratio (AUC: 0.66, 95\% CI: 0.53-0.80). We observed no statistically significant differences between the AUC of any of the fasting indexes (Table 4).

Finally, we validated the score using the baseline evaluation of our MS cohort $(n=9637)$ against HOMA-IR, defining IR as HOMA-IR score $>75$ th percentile $(>3.78$, Supplementary Table 5). Using partial correlation analyses, adjusted for age and sex, we found the highest correlation to HOMA-IR among non-insulin-based indexes for METS-IR $(\rho=-0.568,95 \% \mathrm{CI}$ : -0.554 to $-0.582)$, followed by the TyG-BMI index; as expected, the highest correlation was observed for the QUICKI index. Among non-insulin-based indexes, METS-IR had significantly higher AUCs compared to the TyG index and the TG/HDL ratio $(P<0.001)$; we observed no significant differences between METS-IR and the TyG-BMI index (Fig. 3D and Table 4).

\section{Prediction of incident T2D using baseline METS-IR}

Our validation cohort included 9637 subjects for the baseline evaluation, from which 6144 completed the follow-up evaluation. We observed 331 cases of incident T2D over 14850 accumulated person-years, yielding an incidence rate of 22.3 cases per 1000 person-years, or $5.4 \%$ in an average of 2.42 years of follow-up. Most subjects

Table 3 Linear regression analyses to evaluate the association of METS-IR and subrogates of insulin resistance, adjusted for age, sex and the presence of T2D.

\begin{tabular}{|c|c|c|c|c|c|}
\hline & Beta & Standardized beta & $\boldsymbol{T}$ & $P$-value & $95 \% \mathrm{Cl}$ \\
\hline \multicolumn{6}{|l|}{ Parameter } \\
\hline Fasting insulin & 0.285 & 0.189 & 2.639 & 0.010 & $0.071-3.746$ \\
\hline Intrahepatic fat & 1.652 & 0.267 & 3.455 & 0.001 & $0.704-2.599$ \\
\hline Intravisceral fat & 3.217 & 0.274 & 3.092 & 0.003 & $1.154-5.281$ \\
\hline Subcutaneous fat & 5.892 & 0.88 & 4.309 & $<0.001$ & $3.181-8.603$ \\
\hline
\end{tabular}

95\% Cl, 95\% Confidence interval; METS-IR, Metabolic Score for Insulin Resistance. 

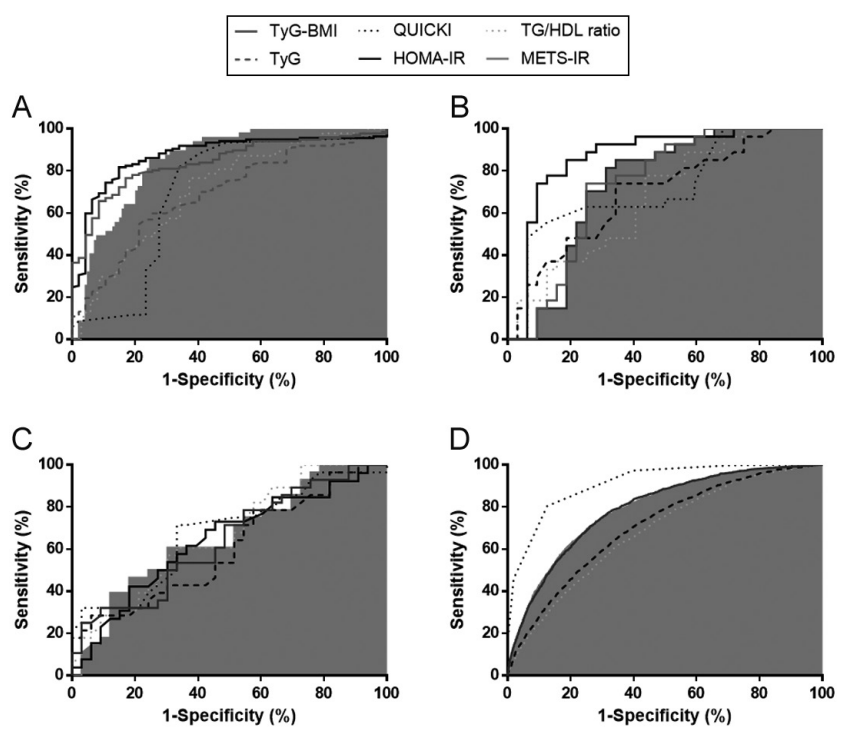

Figure 3

Receiver-operating characteristic (ROC) curves showing the weighed sensitivity and 1-specificity measures of fasting IR surrogates to identify cases of insulin resistance against (A). $M$-value adjusted by fat-free mass as the gold standard with a cut-off point $<25$ th percentile in the combined EHC cohort ( $n=184)$. (B) M-value adjusted by fat-free mass as the gold standard with a cut-off point $<25$ th percentile in the validation EHC cohort $n=59$ ), (C) SI index obtained using MINMOD analyses from modified FSIVGTT using a cut-off of $<5 \mu \mathrm{U} / \mathrm{min} / \mathrm{mL}(n=61)$ and (D) HOMA-IR values $>75$ th percentile in the baseline evaluation of our MS cohort ( $n=9637)$. Shaded area represents area under the ROC curve of METS-IR. HOMA-IR, homeostatic model assessment for IR; METS-IR, Metabolic Score for Insulin Resistance; QUICKI, quantitative insulin sensitivity check index; TG/HDL, Ratio of triglycerides and high-density lipoprotein cholesterol; TyG, TyG index; TyG-BMI, TyG*BMI index.

were female $(66.6 \%)$, with no significant differences in sex between those who developed T2D after follow-up in comparison to those who did not. The mean age of enrolled subjects was $42.63 \pm 10.79$ years, with a significantly higher age for subjects with incident T2D. Subjects who developed T2D had significantly higher BMI, glucose, insulin, triglyceride, total cholesterol along with lower HDL-c levels (Table 5). After follow-up, subjects with incident T2D had a significant increase in fasting glucose and a decrease in triglyceride, total cholesterol, HDL-c and without significant changes in BMI and fasting insulin levels.

Subjects who developed T2D had significantly higher METS-IR scores at baseline in comparison to those who did not (Fig. 4A, $50.2 \pm 10.2$ vs $44.7 \pm 9.2, P<0.001$ ). Both groups had a slight increase in METS-IR scores between visits, which remained significantly larger in subjects with incident T2D. Individuals in the highest METS-IR tercile had significantly higher T2D incidence over time, in comparison to the middle and lower terciles (log-rank test $P<0.001$; Fig. $4 \mathrm{~B}$ ). This observation was confirmed in Cox proportional risks regression analysis, which showed progressively higher risk of incident T2D for the highest (METS-IR >47.86; HR: 2.92, 95\% CI: 1.87-4.55) and middle tercile (METS-IR: 40.16-47.86; HR: 2.38, 95\% CI: 1.51-3.76) in comparison to the lowest tercile, adjusted by family history of T2D, age, sex, smoking, hypertension, physical activity and waist circumference. Using METS-IR quartiles, a score > 50.39 was associated with the highest adjusted risk to develop T2D (HR: 3.91, 95\% CI: 2.25-6.81; Supplementary Table 6).

\section{Discussion}

Here, we report a novel surrogate index to estimate insulin action validated against the EHC. Our model is calculated using fasting measures of glucose, triglycerides and HDL-c along with BMI, which are routinely obtained by primary care physicians, and do not rely on fasting insulin measurements, which are costly and have a high variability according to immunoassay technique utilized (18). METS-IR is a simple, indirect method for the detection of IR that correlates with pathophysiological components of the MS (i.e. intravisceral, intrahepatic and intrapancreatic fat) and is useful for prediction of incident T2D.

The performance of METS-IR was compared against other surrogate IR indexes. The higher correlations with the MFFM were observed for METS-IR, HOMA-IR, QUICKI and the TyG-BMI indexes and were similar to previous reports (19). METS-IR had a good diagnostic performance that was significantly higher than the TyG index and the TG/HDL ratio, but no different than the TyG-BMI index. Likewise, non-insulin-based indexes, particularly METS-IR and the TyG-BMI index, had a better correlation with MFFM than insulin-based indexes. Analyses of MS components have suggested a higher sensitivity for surrogates of obesity to identify adverse metabolic outcomes $(20,21)$. Therefore, the use of BMI in IR estimation might increase the spectrum of explained variability of the model and elucidates the increased correlation and diagnostic performance for both METS-IR and the TyG-BMI index, both of which include anthropometric measurements, against other non-insulinbased fasting surrogates of IR; this is relevant, since obesity is a known modifier of the reliability of insulin and noninsulin-based estimates of IR $(22,23,24)$. 
Table 4 Comparison of areas under the receiver operating characteristic curves for insulin resistance subrogate indexes as compared to the M-value adjusted by fat-free mass between the overall population, subjects with T2D and controls. Data are presented as AUC $(95 \% \mathrm{Cl})$

\begin{tabular}{|c|c|c|}
\hline Index & ${\text { EHC } \text { cohort }^{+}(n=184)}$ & EHC validation ${ }^{+}$cohort $(n=59)$ \\
\hline METS-IR & $0.845(0.783-0.899)$ & $0.738(0.601-0.866)$ \\
\hline HOMA-IR & $0.875(0.812-0.926)$ & $0.868(0.756-0.957)$ \\
\hline QUICKI & $0.702(0.596-0.809)$ * & $0.720(0.518-0.846)$ \\
\hline TyG index & $0.692(0.609-0.771)$ * & $0.692(0.555-0.822)$ \\
\hline TyG-BMI index & $0.841(0.778-0.899)$ & $0.733(0.596-0.863)$ \\
\hline TG/HDL & $0.710(0.626-0.790)$ * & $0.672(0.531-0.806)$ \\
\hline
\end{tabular}

\begin{tabular}{c}
\hline FSIVGTT cohort^ $(n=61)$ \\
\hline $0.669(0.532-0.806)$ \\
$0.645(0.501-0.790)$ \\
$0.681(0.544-0.818)$ \\
$0.632(0.490-0.774)$ \\
$0.640(0.499-0.780)$ \\
$0.663(0.527-0.800)$
\end{tabular}

\begin{tabular}{c}
$\begin{array}{c}\text { Metabolic syndrome } \\
\text { cohort }^{8}(n=9637)\end{array}$ \\
\hline $0.800(0.789-0.811)$ \\
- \\
$0.944(0.940-0.949)$ * \\
$0.715(0.703-0.728)^{*}$ \\
$0.800(0.790-0.811)$ \\
$0.690(0.677-0.703)$ *
\end{tabular}

${ }^{*} P<0.01$ against METS-IR in non-parametric ROC AUC comparison, ${ }^{+} \mathrm{IR}$ defined as MFFM $<25$ th percentile, $\wedge I R$ defined as $\mathrm{SI}$ index $<5 \mu \mathrm{U} / \mathrm{min} / \mathrm{mL},{ }^{\&} \mathrm{R}$ defined ad HOMA-IR values $>75$ th percentile.

95\% Cl, 95\% Confidence interval; AUC, Area under the curve; HOMA-IR, homeostatic model assessment for IR; IR, Insulin resistance; METS-IR, Metabolic Score for Insulin Resistance; QUICKI, quantitative insulin sensitivity check index; TG/HDL, Ratio of triglycerides and high-density lipoprotein cholesterol; TyG, TyG index; TyG-BMI, TyG*BMI index; T2D, Type 2 diabetes mellitus.

METS-IR demonstrated significant correlations with visceral, intrahepatic and intrapancreatic fat content, known pathophysiological components of both IR and MS. Ectopic fat accumulation in muscle and liver tissue has been studied as a mechanism for the development of IR $(25,26)$; several studies have linked intrahepatic and intrapancreatic fat accumulation with IR $(27,28,29,30)$. Intrahepatic fat accumulation has also been linked to the development of hepatic IR, which significantly alters glucose and lipid homeostasis $(31,32)$. This translates into hyperglycemia, impaired lipemia and increases in body weight, all of which are mechanisms evaluated by our index (33). Evaluation of at-risk individuals using METS-IR would allow identification of pathophysiological alterations of IR, sparing the cost and variability of fasting insulin measurements.

Validation of the index against the MFFM was carried out combining the discovery sample with a set of obese individuals in which EHC data were available. In addition, we extended the validation to a second cohort of individuals with normal BMI, using as a gold standard a modified FSIVGTT with minimal model analysis to assess insulin sensitivity. This approach is reasonable, since minimal model approaches yield measures of insulin sensitivity that adequately correlate with estimations made by clamp techniques (34). Despite obtaining a lower correlation with SI than the observed with MFFM, METS-IR had a good diagnostic performance to identify cases of IR against other fasting surrogates of IR in this second cohort. Finally, we performed a third validation against HOMA-IR in a large cohort, in which the observations from validation against the other two measures of insulin sensitivity were replicated. Thus, METS-IR had good diagnostic performance in all three cohorts and was validated against three different methods to estimate insulin action.

IR is known to increase the risk for the development of dyslipidemia, hypertension, coronary artery disease and, particularly T2D $(35,36)$. Fasting IR indexes, including QUICKI, HOMA-IR, TyG index and the TG/HDL ratio have all been shown to predict incident T2D $(37,38,39,40)$. In our study, individuals in the highest percentile of METS-IR

Table 5 Anthropometric and laboratory measures for subjects with and without incident type 2 diabetes after 2-year follow-up in the validation cohort.

\begin{tabular}{l} 
Parameter \\
\hline METS-IR \\
Fasting glucose $(\mathrm{mg} / \mathrm{dL})$ \\
BMI $\left(\mathrm{kg} / \mathrm{m}^{2}\right)$ \\
Triglycerides $(\mathrm{mg} / \mathrm{dL})$ \\
Fasting insulin $(\mu \mathrm{l} / \mathrm{mL})$ \\
Total cholesterol $(\mathrm{mg} / \mathrm{dL})$ \\
HDL-c $(\mathrm{mg} / \mathrm{dL})$
\end{tabular}

\begin{tabular}{ccc}
\hline \multicolumn{2}{c}{ Control } \\
\cline { 1 - 1 } \cline { 1 - 1 } Baseline & Follow-up \\
\hline $44.67 \pm 9.22$ & & $45.71 \pm 10.00$ \\
$85.35 \pm 10.45$ & & $85.53 \pm 11.89$ \\
$28.65 \pm 4.58$ & & $28.74 \pm 4.66$ \\
$187.90 \pm 141.26$ & & $174.20 \pm 112.42$ \\
$11.75 \pm 7.64$ & & $12.11 \pm 10.14$ \\
$205.76 \pm 40.94$ & & $197.78 \pm 40.07$ \\
$44.81 \pm 11.69$ & & $41.71 \pm 12.11$ \\
\hline
\end{tabular}

$\begin{array}{r}\hline \boldsymbol{P} \text {-value* } \\ \hline<0.001 \\ 0.227 \\ 0.001 \\ <0.001 \\ 0.007 \\ <0.001 \\ <0.001 \\ \hline\end{array}$

\begin{tabular}{c}
\hline Incident d \\
\hline Baseline \\
\hline $50.23 \pm 10.16$ \\
$97.47 \pm 13.42$ \\
$31.01 \pm 5.19$ \\
$226.93 \pm 154.87$ \\
$15.82 \pm 10.30$ \\
$211.17 \pm 40.69$ \\
$42.41 \pm 10.97$ \\
\hline
\end{tabular}

\begin{tabular}{l} 
diabetes \\
\hline Follow-up \\
\hline $51.04 \pm 10.90$ \\
$112.69 \pm 38.41$ \\
$30.79 \pm 5.18$ \\
$207.59 \pm 121.16$ \\
$17.26 \pm 15.37$ \\
$202.69 \pm 39.33$ \\
$40.20 \pm 10.88$
\end{tabular}

\begin{tabular}{r}
\hline P-value* \\
\hline 0.021 \\
$<0.001$ \\
0.061 \\
0.020 \\
0.083 \\
$<0.001$ \\
$<0.001$
\end{tabular}

* $P$-value for paired comparison between baseline and follow-up

BMI, Body mass index; HDL-c, High-density lipoprotein cholesterol; LDL-c, Low-density lipoprotein cholesterol; METS-IR, Metabolic Score for Insulin Resistance. 
A
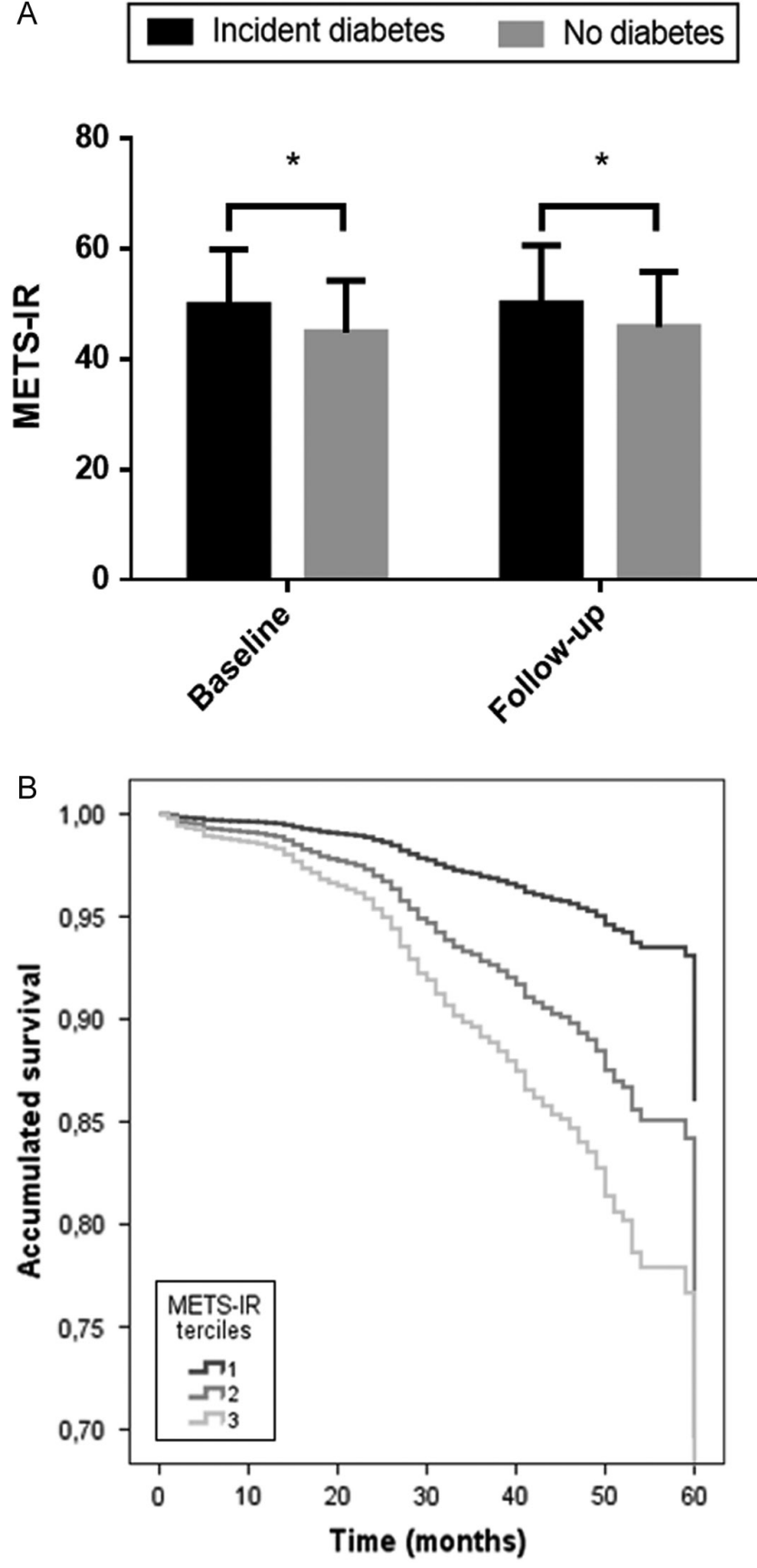

Figure 4

(A) Comparison of mean METS-IR values at baseline and follow-up between subjects with and without incident T2D. As seen in the figure both at baseline and follow-up mean METS-IR was significantly different between subjects with and without incident T2D $(P<0.001)$. (B) Kaplan-Meier survival curves comparing T2D incidence between METS-IR terciles during a two-year follow-up period $(P<0.001$ for log-rank test). score had a 3.9-fold increase in the risk of incident T2D after follow-up, compared to the lowest METS-IR quartile. In addition, individuals who developed T2D had higher baseline METS-IR scores and the risk of incident T2D was progressively higher for increasing METS-IR percentiles scores, an effect that was modulated by age. Application of METS-IR is feasible and reliable to identify subjects at-risk of developing T2D, which makes it a useful tool for application by primary care physicians, considering the practicality of its measurements and the pathophysiological correlations with components of MS and IR.

Our study had some strengths and limitations. METS-IR was validated against MFFM, the gold standard for assessing insulin action without the confounding effect of changes in body composition; normalization of M-values by FFM is a useful technique and reduces underestimation resulting from gender-related differences in fat mass compared to adjustment for body weight (41). METS-IR was validated in a group of patients with the clinical characteristics in which the estimation of insulin action is most likely to be clinically useful. Furthermore, it was validated also against the SI index, obtained with the minimal model approach. The evaluation of our index went beyond the assessment of the insulin action; it included also metabolic comorbidities and the ability to predict incident T2D. The limitations to be acknowledged include a relatively small sample size in the discovery sample and a small number of lean and healthy individuals in the validation sample. Further, we performed validation against the MFFM in an overlapping population with the discovery sample, which could lead to overestimation of diagnostic performance; this issue is common in the development of a surrogate index of insulin action due to the complexity and cost of the EHC. To account for these limitations, we performed validation against other estimators of insulin action and estimated confidence intervals using bootstrap methods; finally, we could replicate the results from the validation against MFFM. In addition, most studies comparing indirect fasting IR indexes use as the M-value adjusted by total body weight as the gold standard, while we used the MFFM, which makes comparisons with results from other studies less feasible. Lastly, because nearly half of the subjects evaluated in the discovery population had T2D, hyperinsulinemia during the one-stage EHC might not completely suppress hepatic glucose production, which might underestimate M-values for individuals with T2D (42); further, correlation with the MFFM and evaluation of ectopic fat accumulation across METS-IR percentiles had to be adjusted by T2D, age and sex, which leaves the possibility of residual confounding. 
In conclusion, METS-IR is a novel score, which combines non-insulin fasting laboratory values and anthropometric measurements easily obtained during a primary care evaluation to evaluate insulin sensitivity and detect IR cases. Our index has a good correlation with the MFFM obtained from the EHC, ectopic fat accumulation and fasting insulin levels, which makes it a reliable indicator of overall IR. Furthermore, METS-IR displays a good predictive capacity to detect individuals at risk of developing T2D, which poses it as a complementary tool to conventional clinical predictors for the development of T2D. Therefore, METS-IR is a promising score to evaluate cardiometabolic risk, which makes it a useful tool for primary care physicians as a routine screening tool for metabolic health.

\section{Supplementary data}

This is linked to the online version of the paper at https://doi.org/10.1530/ EJE-17-0883.

\section{Declaration of interest}

The authors declare that there is no conflict of interest that could be perceived as prejudicing the impartiality of this study.

\section{Funding}

This research did not receive any specific grant from any funding agency in the public, commercial or not-for-profit sector.

\section{Author contribution statement}

Research idea and study design: O Y B C, I C B, P A V, C A A S; data acquisition: P A V, D G V, T V R, A R R, D S L, D M O, O A C, M R S G, A J M, L M H; data analysis/interpretation: O Y B C, P A V, C A A S; statistical analysis: O Y B C, A V V; manuscript drafting: O Y B C, P A V, C A A S, A $V$ V; supervision or mentorship: C A A S, P A V. Each author contributed important intellectual content during manuscript drafting or revision and accepts accountability for the overall work by ensuring that questions pertaining to the accuracy or integrity of any portion of the work are appropriately investigated and resolved.

\section{Acknowledgments}

All authors approved the submitted version. All the authors would like to thank the staff of the Endocrinology and Metabolism Department for all their support, particularly to Luz Elizabeth Guillen-Pineda, Maria Del Carmen Moreno-Villatoro and Adriana Cruz-Lopez. They are thankful to the study volunteers for all their work and support throughout the realization of the study.

\section{References}

1 Gastaldelli A1, Gaggini M \& DeFronzo RA. Role of adipose tissue insulin resistance in the natural history of type 2 diabetes: results from the San Antonio Metabolism Study. Diabetes 201766 815-822. (https://doi.org/10.2337/db16-1167)

2 Bello-Chavolla OY, Rojas-Martinez R, Aguilar-Salinas CA \& Hernández-Avila M. Epidemiology of diabetes mellitus in Mexico. Nutrition Reviews 201775 (Supplement 1) 4-12. (https://doi. org/10.1093/nutrit/nuw030)
3 DeFronzo RA, Tobin JD \& Andres R. Glucose clamp technique: a method for quantifying insulin secretion and resistance. American Journal of Physiology 1979237 E214-E223. (https://doi. org/10.1152/ ajpendo.1979.237.3.E214)

4 Roberts LD, Koulman A \& Griffin JL. Towards metabolic biomarkers of insulin resistance and type 2 diabetes: progress from the metabolome. Lancet Diabetes and Endocrinology 20142 65-75. (https://doi.org/10.1016/S2213-8587(13)70143-8)

5 Borai A, Livingstone C \& Ferns GA. The biochemical assessment of insulin resistance. Annals of Clinical Biochemistry 200744 324-342. (https://doi.org/10.1258/000456307780945778)

6 Simental-Mendía LE, Rodríguez-Morán M \& Guerrero-Romero F. The product of fasting glucose and triglycerides as surrogate for identifying insulin resistance in apparently healthy subjects. Metabolic Syndrome and Related Disorders 20086 299-304. (https://doi. org/10.1089/met.2008.0034)

7 Abbasi F \& Reaven GM. Comparison of two methods using plasma triglyceride concentration as a surrogate estimate of insulin action in nondiabetic subjects: triglycerides $\times$ glucose versus triglyceride/ high-density lipoprotein cholesterol. Metabolism 201160 1673-1676. (https://doi.org/10.1016/j.metabol.2011.04.006)

8 Bastard JP, Vandernotte JM, Faraj M, Karelis AD, Messier L, Malita FM, Garrel D, Prud'homme D \& Rabasa-Lhoret R. Relationship between the hyperinsulinemic-euglycaemic clamp and a new simple index assessing insulin sensitivity in overweight and obese postmenopausal women. Diabetes and Metabolism 2007 33 261-268. (https://doi.org/10.1016/j.diabet.2007.02.004)

9 Borai A, Livingstone C, Kaddam I \& Ferns G. Selection of the appropriate method for the assessment of insulin resistance. BMC Medical Research Methodology 201111 158. (https://doi. org/10.1186/1471-2288-11-158)

10 Dalla Man C, Piccinini F, Basu R, Basu A, Rizza RA \& Cobelli C. Modeling hepatic insulin sensitivity during a meal: validation against the euglycemic hyperinsulinemic clamp. American Journal of Physiology-Endocrinology and Metabolism 2013304 E819-E825. (https://doi.org/10.1152/ajpendo.00482.2012)

11 Wong VW, Chu WC, Wong GL, Chan RS, Chim AM, Ong A, Yeung DK, Yiu KK, Chu SH, Woo J et al. Prevalence of non-alcoholic fatty liver disease and advanced fibrosis in Hong Kong Chinese: a population study using proton-magnetic resonance spectroscopy and transient elastography. Gut 201261 409-415. (https://doi. org/10.1136/gutjnl-2011-300342)

12 Matthews DR, Hosker JP, Rudenski AS, Naylor BA, Treacher DF $\&$ Turner RC. Homeostasis model assessment: insulin resistance and beta-cell function from fasting plasma glucose and insulin concentrations in man. Diabetologia 198528 412-419. (https://doi. org/10.1007/BF00280883)

13 Katz A, Nambi SS, Mather K, Baron AD, Follmann DA, Sullivan G \& Quon MJ. Quantitative insulin sensitivity check index: a simple, accurate method for assessing insulin sensitivity in humans. Journal of Clinical Endocrinology and Metabolism 200085 2402-2410. (https:// doi.org/10.1210/jcem.85.7.6661)

14 Kannel WB, Vasan RS, Keyes MJ, Sullivan LM \& Robins SJ. Usefulness of the triglyceride-high-density lipoprotein versus the cholesterolhigh-density lipoprotein ratio for predicting insulin resistance and cardiometabolic risk (from the Framingham Offspring Cohort). American Journal of Cardiology 2008101 497-501. (https://doi. org/10.1016/j.amjcard.2007.09.109)

15 Er LK, Wu S, Chou HH, Hsu LA, Teng MS, Sun YC \& Ko YL. Triglyceride glucose-body mass index is a simple and clinically useful surrogate marker for insulin resistance in nondiabetic individuals. PLOS ONE 201611 e0149731. (https://doi.org/10.1371/journal.pone.0149731)

16 Robin X, Turck N, Hainard A, Tiberti N, Lisacek F, Sanchez JC \& Müller M. pROC: an open-source package for R and S+ to analyze and compare ROC curves. BMC Bioinformatics 201112 77. (https:// doi.org/10.1186/1471-2105-12-77) 
17 Lopez-Raton M, Rodriguez-Alvarez MX, Cadarso-Suarez C \& GudeSampedro F. OptimalCutpoints: an R package for selecting optimal cutpoints in diagnostic tests. Journal of Statistical Software $2014 \mathbf{6 1}$ 1-36. (https://doi.org/10.18637/jss.v061.i08)

18 Marcovina S, Bowsher RR, Miller WG, Staten M, Myers G, Caudill SP, Campbell SE \& Steffes MW. Standardization of insulin immunoassays: report of the American Diabetes Association Workgroup. Clinical Chemistry 200753 711-716. (https://doi. org/10.1373/clinchem.2006.082214)

19 Otten J, Ahrén B \& Olsson T. Surrogate measures of insulin sensitivity vs the hyperinsulinaemic-euglycaemic clamp: a metaanalysis. Diabetologia 201457 1781-1788. (https://doi.org/10.1007/ s00125-014-3285-x)

20 Almeda-Valdes P, Herrera-Mercadillo RJ, Aguilar-Salinas CA, Uribe M \& Méndez-Sánchez $\mathrm{N}$. The role of diet in patients with metabolic syndrome. Current Medicinal Chemistry 2017 24. (https://doi.org/10.2 174/0929867324666170518095316)

21 Murguía-Romero M, Jiménez-Flores JR, Sigrist-Flores SC, TapiaPancardo DC, Jiménez-Ramos A, Méndez-Cruz AR \& VillalobosMolina R. Prevalence of metabolic syndrome in young mexicans: a sensitivity analysis on its components. Nutricion Hospitalaria 2015 32 189-195. (https://doi. org/10.3305/nh.2015.32.1.9031)

22 Lee SH, Han K, Yang HK, Kim MK, Yoon KH, Kwon HS \& Park YM. Identifying subgroups of obesity using the product of triglycerides and glucose: the Korea National Health and Nutrition Examination Survey, 2008-2010. Clinical Endocrinology 201582 213-220. (https:// doi.org/10.1111/cen.12502)

23 Jones CN, Abbasi F, Carantoni M, Polonsky KS \& Reaven GM. Roles of insulin resistance and obesity in regulation of plasma insulin concentrations. American Journal of Physiology-Endocrinology and Metabolism 2000278 E501-E508. (https://doi.org/10.1152/ ajpendo.2000.278.3.E501)

24 Kim SH, Abbasi F \& Reaven GM. Impact of degree of obesity on surrogate estimates of insulin resistance. Diabetes Care 200427 1998-2002. (https://doi.org/10.2337/diacare.27.8.1998)

25 Roden M, Price TB, Perseghin G, Petersen KF, Rothman DL, Cline GW \& Shulman GI. Mechanism of free fatty acid-induced insulin resistance in humans. Journal of Clinical Investigation $1996 \mathbf{9 7}$ 2859-2865. (https://doi.org/10.1172/JCI118742)

26 Shulman GI. Ectopic fat in insulin resistance, dyslipidemia, and cardiometabolic disease. New England Journal of Medicine 2014371 1131-1141. (https://doi.org/10.1056/NEJMra1011035)

27 Kato K, Takamura T, Takeshita Y, Ryu Y, Misu H, Ota T, Tokuyama K, Nagasaka S, Matsuhisa M, Matsui O et al. Ectopic fat accumulation and distant organ-specific insulin resistance in Japanese people with nonalcoholic fatty liver disease. PLoS ONE 20149 e92170. (https:// doi.org/10.1371/journal.pone.0092170)

28 Lomonaco R, Ortiz-Lopez C, Orsak B, Webb A, Hardies J, Darland C, Finch J, Gastaldelli A, Harrison S, Tio F et al. Effect of adipose tissue insulin resistance on metabolic parameters and liver histology in obese patients with nonalcoholic fatty liver disease. Hepatology 2012 55 1389-1397. (https://doi.org/10.1002/hep.25539)

29 Singh RG, Yoon HD, Poppitt SD, Plank LD \& Petrov MS. Ectopic fat accumulation in the pancreas and its biomarkers: a systematic review and meta-analysis. Diabetes/Metabolism Research and Reviews 201733 e2918. (https://doi.org/10.1002/dmrr.2918)

30 Wong VW, Wong GL, Yeung DK, Abrigo JM, Kong AP, Chan RS, Chim AM, Shen J, Ho CS, Woo J et al. Fatty pancreas, insulin resistance, and $\beta$-cell function: a population study using fat-water magnetic resonance imaging. American Journal of Gastroenterology 2014109 589-597. (https://doi.org/10.1038/ajg.2014.1)

31 Mehta SR, Godsland IF, Thomas EL, Pavitt DV, Morin SX, Bell JD, Taylor-Robinson SD \& Johnston DG. Intrahepatic insulin exposure, intrahepatocellular lipid and regional body fat in nonalcoholic fatty liver disease. Journal of Clinical Endocrinology and Metabolism 201297 2151-2159. (https://doi.org/10.1210/jc.2011-2430)

32 Wueest S, Item F, Lucchini FC, Challa TD, Müller W, Blüher M $\&$ Konrad D. Mesenteric fat lipolysis mediates obesity-associated hepatic steatosis and insulin resistance. Diabetes 201665 140-148. (https://doi.org/10.2337/db15-0941)

33 Shanik MH, Xu Y, Skrha J, Dankner R, Zick Y \& Roth J. Insulin resistance and hyperinsulinemia: is hyperinsulinemia the cart or the horse? Diabetes Care 200831 (Supplement 2) S262-S268. (https://doi. org/10.2337/dc08-s264)

34 Coates PA, Luzio SD, Brunel P \& Owens DR. Comparison of estimates of insulin sensitivity from minimal model analysis of the insulinmodified frequently sampled intravenous glucose tolerance test and the isoglycemic hyperinsulinemic clamp in subjects with NIDDM. Diabetes 199544 631-635. (https://doi.org/10.2337/diab.44.6.631)

35 Wang F, Han L \& Hu D. Fasting insulin, insulin resistance and risk of hypertension in the general population: a meta-analysis. Clinica Chimica Acta 2017464 57-63. (https://doi.org/10.1016/j.cca.2016.11.009)

36 Reaven G. Insulin resistance and coronary heart disease in nondiabetic individuals. Arteriosclerosis, Thrombosis, and Vascular Biology 201232 1754-1759. (https://doi.org/10.1161/ ATVBAHA.111.241885)

37 Lee $\mathrm{CH}$, Shih AZ, Woo YC, Fong CH, Leung OY, Janus E, Cheung BM \& Lam KS. Optimal cut-offs of Homeostasis Model Assessment of Insulin Resistance (HOMA-IR) to identify dysglycemia and type 2 diabetes mellitus: a 15-year prospective study in Chinese. PLOS ONE 201611 e0163424. (https://doi. org/10.1371/journal.pone.0163424)

38 Vanhala P, Vanhala M, Kumpusalo E \& Keinänen-Kiukaanniemi S. The quantitative insulin sensitivity check index QUICKI predicts the onset of type 2 diabetes better than fasting plasma insulin in obese subjects: a 5-year follow-up study. Journal of Clinical Endocrinology and Metabolism 200287 5834-5837. (https://doi.org/10.1210/ jc.2002-020591)

39 Vega GL, Barlow CE, Grundy SM, Leonard D \& DeFina LF. Triglyceride-to-high-density-lipoprotein-cholesterol ratio is an index of heart disease mortality and of incidence of type 2 diabetes mellitus in men. Journal of Investigative Medicine 201462 345-349. (https:// doi.org/10.2310/JIM.0000000000000044)

40 Lee SH, Kwon HS, Park YM, Ha HS, Jeong SH, Yang HK, Lee JH, Yim HW, Kang MI, Lee WC et al. Predicting the development of diabetes using the product of triglycerides and glucose: the Chungju Metabolic Disease Cohort (CMC) study. PLoS ONE 20149 e90430. (https://doi.org/10.1371/journal.pone.0090430)

41 Bokemark L, Frödén A, Attvall S, Wikstrand J \& Fagerberg B. The euglycemic hyperinsulinemic clamp examination: variability and reproducibility. Scandinavian Journal of Clinical and Laboratory Investigation 200060 27-36. (https://doi. org/10.1080/00365510050185010)

42 Campbell PJ, Mandarino LJ \& Gerich JE. Quantification of the relative impairment in actions of insulin on hepatic glucose production and peripheral glucose uptake in non-insulin-dependent diabetes mellitus. Metabolism 198837 15-21. (https://doi. org/10.1016/0026-0495(88)90023-6)
Received 24 October 2017

Revised version received 18 February 2018

Accepted 12 March 2018 